ŁukAsz StetTner (Warszawa)

\title{
DISCRETE TIME RISK SENSITIVE PORTFOLIO OPTIMIZATION WITH CONSUMPTION AND PROPORTIONAL TRANSACTION COSTS
}

Abstract. Risk sensitive and risk neutral long run portfolio problems with consumption and proportional transaction costs are studied. Existence of solutions to suitable Bellman equations is shown. The asymptotics of the risk sensitive cost when the risk factor converges to 0 is then considered. It turns out that optimal strategies are stationary functions of the portfolio (portions of the wealth invested in assets) and of economic factors. Furthermore an optimal portfolio strategy for a risk neutral control problem is nearly optimal for a risk sensitive portfolio cost functional with risk factor close to 0 .

1. Introduction. Assume we are given a discrete time market with $m$ risky assets. Denote by $S_{i}(t)$ the price of the $i$ th asset at time $t$. Assume that

$$
\frac{S_{i}(t+1)}{S_{i}(t)}=\zeta_{i}(z(t+1), \xi(t+1))
$$

where $(z(t)) \in \mathbb{R}^{d}$ is a Markov process with transition operator $P(z(t), d y)$ describing the evolution of economic factors, $(\xi(t))$ stands for a sequence of i.i.d. random variables, independent of $(z(t))$, and $\zeta$ is a given positive function such that the mapping $z \mapsto \zeta(z, \xi)$ is continuous for $\xi>0$. Denote by $X^{-}(t)$ the wealth process at time $t$ before consumption and possible transactions, and by $X(t)$ the wealth process after possible transactions. Let $\pi_{i}^{-}(t)$ be the portion of the wealth process invested in the $i$ th asset at time $t$ before consumption and possible transactions, and $\pi_{i}(t)$ the portion of the wealth located in the $i$ th asset after transactions at time $t$. We shall say that $\pi(t)=\left(\pi_{1}(t), \ldots, \pi_{m}(t)\right)^{T}$ (where $T$ stands for transpose) and similarly

2000 Mathematics Subject Classification: Primary 93E20; Secondary 60J05, 93C55.

Key words and phrases: risk sensitive control, discrete time Markov processes, wealth process, optimal portfolio, Bellman equation.

Research supported by MNiI grant 1 P03A 01328. 
$\pi^{-}(t)$ form portfolios at time $t$ after and before consumption and possible transactions. Denote by $\alpha(t)$ the portion of capital consumed at time $t$. Let $S^{0}=\left\{\left(\nu_{1}, \ldots, \nu_{m}\right)^{T}: \nu_{i} \geq 0, \sum_{i=1}^{m} \nu_{i} \leq 1\right\}$ and $S=\left\{\left(\nu_{1}, \ldots, \nu_{m}\right)^{T} \in S^{0}:\right.$ $\left.\sum_{i=1}^{m} \nu_{i}=1\right\}$. For given $\pi \in S^{0}$ let

$$
g(\pi)=\left(g_{1}(\pi), \ldots, g_{m}(\pi)\right)^{T}, \quad \text { where } \quad g_{i}(\pi)=\frac{\pi_{i}}{\sum_{j=1}^{m} \pi_{j}} .
$$

After a change of portfolio from $\pi$ to $\pi^{\prime}$ the wealth $X$ is diminished by $c(\widehat{\pi}-\pi) X$, where $\widehat{\pi}$ is a certain element of $S^{0}$ (we shall see in Lemma 1 that it is unique) such that $\pi^{\prime}=g(\widehat{\pi})$ and for $\nu \in S^{0}-S^{0}$ (the algebraic difference)

$$
c(\nu)=\sum_{i=1}^{m} c_{i}^{1}\left(\nu_{i}\right)^{+}+\sum_{i=1}^{m} c_{i}^{2}\left(\nu^{i}\right)^{-}
$$

with $0<c_{i}^{1}, c_{i}^{2}<1$. Given a portfolio $\pi$ and wealth $X$ we can consume a portion $\alpha$ of the portfolio and change the portfolio to $\pi^{\prime}$. Since our consumption has to be compensated by suitable selling of assets there should exist $\widehat{\pi}$ such that

$$
X(c(\widehat{\pi}-\pi)+\alpha)=X-X \sum_{i=1}^{m} \widehat{\pi}_{i}
$$

and $g(\widehat{\pi})=\pi^{\prime}$. Consequently, given $\pi$ we can consume a portion $\alpha$ and afterwards choose $\pi^{\prime}$ if and only if there is $\widehat{\pi} \in S^{0}$ such that

$$
\sum_{i=1}^{m} \widehat{\pi}_{i}+c(\widehat{\pi}-\pi)+\alpha=1
$$

where

$$
\pi^{\prime}=g(\widehat{\pi}) .
$$

In general for given $\pi$ and $\alpha$, not all $\pi^{\prime} \in S$ are admissible. In what follows we shall assume that we are allowed to consume only a part of the available wealth, i.e. there is a $\Lambda>\sup _{\pi \in S} c(-\pi)$ such that

$$
0 \leq \alpha \leq 1-\Lambda \text {. }
$$

Given $\pi, \pi^{\prime} \in S$ and $\alpha \in[0, \Lambda]$ define the function

$$
F_{\alpha}^{\pi, \pi^{\prime}}(\delta):=\delta+c\left(\delta \pi^{\prime}-\pi\right)+\alpha .
$$

LEMma 1. There is a unique continuous function $e: S \times S \times[0, \Lambda] \rightarrow[0,1]$ such that for $\pi, \pi^{\prime} \in S$ and $\alpha \in[0, \Lambda]$ we have

$$
F_{\alpha}^{\pi, \pi^{\prime}}\left(e\left(\pi, \pi^{\prime}, \alpha\right)\right)=1 .
$$

Furthermore e is bounded away from 0. 
Proof. Notice that the function $F_{\alpha}^{\pi, \pi^{\prime}}$ is continuous, strictly increasing and $F_{\alpha}^{\pi, \pi^{\prime}}(0)=c(-\pi)+\alpha \leq 1$, while $F_{\alpha}^{\pi, \pi^{\prime}}(1)=1+c\left(\pi^{\prime}-\pi\right)+\alpha \geq 1$. Therefore there is a unique $e\left(\pi, \pi^{\prime}, \alpha\right)$ satisfying (8). It remains to show continuity of $e$. Let $\pi^{n}, \pi^{\prime n}, \alpha^{n}$ be such that $\pi^{n} \rightarrow \pi, \pi^{\prime n} \rightarrow \pi^{\prime}, \alpha^{n} \rightarrow \alpha$. Since $S$ is compact there are subsequences, for simplicity denoted by $n$, such that $e\left(\pi^{n}, \pi^{\prime n}, \alpha^{n}\right) \rightarrow a$ as $n \rightarrow \infty$. If $a \neq e\left(\pi, \pi^{\prime}, \alpha\right)$, then by continuity of $F$ we have $1=F_{\alpha^{n}}^{\pi^{n}, \pi^{\prime n}}\left(e\left(\pi^{n}, \pi^{\prime n}, \alpha^{n}\right)\right) \rightarrow F_{\alpha}^{\pi, \pi^{\prime}}(a)$, and consequently $a=$ $e\left(\pi, \pi^{\prime}, \alpha\right)$, a contradiction. Assume now that $e$ is not bounded away from 0 . Then there are $\pi, \pi^{\prime} \in S$ and $\alpha \in[0, \Lambda]$ such that $e\left(\pi, \pi^{\prime}, \alpha\right)=0$. Therefore $F_{\alpha}^{\pi, \pi^{\prime}}(0)=c(-\pi)+\alpha=1$, which contradicts $(6)$.

Consequently, given an initial wealth process $X^{-}(t)$ and portfolio $\pi^{-}(t)$ at time $t$ under (6) we choose a consumption portion $\alpha(t)$ from $[0, \Lambda]$ and any post transaction portfolio $\pi(t) \in S$. Then, as a result of transaction costs and consumption our wealth process is diminished to $X(t)$, where following (3) and (4) we have

$$
X(t)=e\left(\pi^{-}(t), \pi(t), \alpha(t)\right) X^{-}(t) .
$$

Furthermore

$$
\begin{aligned}
X^{-}(t+1) & =\sum_{i=1}^{m} \frac{\pi_{i}(t) X(t)}{S_{i}(t)} S_{i}(t+1) \\
& =X(t) \sum_{i=1}^{m} \pi_{i}(t) \zeta_{i}(z(t+1), \xi(t+1)) \\
& :=X(t) \pi(t)^{T} \zeta(z(t+1), \xi(t+1)) .
\end{aligned}
$$

and

$$
\pi^{-}(t+1)=g(\pi(t) \diamond \zeta(z(t+1), \xi(t+1))
$$

with

$$
(\pi(t) \diamond \zeta(z(t+1), \xi(t+1)))_{i}:=\pi_{i}(t) \zeta_{i}(z(t+1), \xi(t+1)) .
$$

Therefore for $t=1,2, \ldots$,

$$
X^{-}(t)=X^{-}(0) \prod_{n=0}^{t-1} e\left(\pi^{-}(n), \pi(n), \alpha(n)\right) \pi(n)^{T} \zeta(z(n+1), \xi(n+1)) .
$$

In this paper we are interested in maximizing the following two cost functionals: the risk sensitive long run cost

$$
\begin{aligned}
& J_{X^{-}, z, \pi^{-}}^{\gamma}(\alpha(n), \pi(n)) \\
&:=\liminf _{t \rightarrow \infty} \frac{1}{\gamma t} \ln E_{X^{-}, z, \pi^{-}}\left\{\left(X^{-}(t)\right)^{\gamma} \prod_{n=0}^{t-1}(h(\alpha(n)))^{\gamma}\right\}
\end{aligned}
$$

and the risk neutral long run cost 


$$
\begin{aligned}
& J_{X^{-}, z, \pi^{-}}(\alpha(n), \pi(n)) \\
& :=\liminf _{t \rightarrow \infty} \frac{1}{t} E_{X^{-}, z, \pi^{-}}\left\{\ln X(t)+\sum_{n=0}^{t-1} \ln h(\alpha(n))\right\}
\end{aligned}
$$

over all admissible, i.e. adapted to available information, sequences $\alpha(n) \in$ $[0, \Lambda]$ and $\pi(n) \in S$, where $h$ is a given continuous function taking positive values, expressing the correction to our terminal utility function that corresponds to the consumption rate, and $\gamma$ is a negative risk factor. Notice following [1] and [2] that the cost functional $J^{\gamma}$ measures average growth of portfolio plus its variance with a negative weight $\gamma$. Moreover by (12) the cost functionals (13) and (14) are of the form

$$
\begin{aligned}
J_{X^{-}, z, \pi^{-}}^{\gamma}(\alpha(n), \pi(n))=\liminf _{t \rightarrow \infty} \frac{1}{\gamma t} \ln E_{X^{-}, z, \pi^{-}}\left\{\prod_{n=0}^{t-1}[h(\alpha(n))\right. \\
\left.\left.\times e\left(\pi^{-}(n), \pi(n), \alpha(n)\right) \pi(n)^{T} \zeta(z(n+1), \xi(n+1))\right]^{\gamma}\right\}
\end{aligned}
$$

and

$$
\begin{aligned}
J_{X^{-}, z, \pi^{-}}(\alpha(n), \pi(n))=\liminf _{t \rightarrow \infty} \frac{1}{t} E_{X^{-}, z, \pi^{-}}\left\{\sum_{n=0}^{t-1} \ln [h(\alpha(n))\right. \\
\left.\left.\times e\left(\pi^{-}(n), \pi(n), \alpha(n)\right) \pi(n)^{T} \zeta(z(n+1), \xi(n+1))\right]\right\} .
\end{aligned}
$$

Risk sensitive portfolio optimization has been the subject of intensive studies in a number of papers (see [1], [2], [7], [10] and [13]). The case with proportional transaction costs was studied in [2] and [13]. In [2] the result was formulated under the assumption of the existence of a nice solution to a suitable Bellman equation. In [13] a more general model was considered in which the factors were allowed to depend on the same random disturbance $(\xi(t))$. However, consumption was not taken into account and a technical assumption concerning an obligatory diversification of portfolio was imposed.

In this paper we allow consumption and have no restrictions on the choice of portfolio. On the other hand, we assume that the factors are independent of the disturbances $(\xi(t))$. Risk neutral control with proportional transaction costs was also considered in [9], where the case with $(\zeta(t))$ of the form of a sequence of i.i.d. random variables (without economic factors $(z(t))$ ) was studied. Some remarks concerning relaxation of ergodic assumptions imposed on the factor process $(z(t))$ as well as allowing the same disturbances in the evolution of asset prices and economic factors are given in Section 5.

2. Risk neutral Bellman equation. We shall assume that

$$
\sup _{z, z^{\prime} \in \mathbb{R}^{d}} \sup _{A \in \mathcal{B}\left(\mathbb{R}^{d}\right)}\left(P(z, A)-P\left(z^{\prime}, A\right)\right)=: \kappa<1
$$


and

$$
\mathbb{R}^{d} \times S \ni(z, \pi) \mapsto f(z, \pi):=E\left[\ln \left(\pi^{T} \zeta(z, \xi(1))\right)\right]
$$

is continuous and bounded.

We can now solve the Bellman equation corresponding to the risk neutral cost functional (14). As we show below the optimal value of this cost functional is a function of the current value of the portfolio process $\pi^{-}(n)$ and the factor process $(z(n))$ and does not depend explicitly on the wealth process. We have the following

THEOREM 1. Assume that the transition operator $P$ is continuous in variation topology, i.e. for $x_{n} \rightarrow x$ the measures $P\left(x_{n}, \cdot\right)$ converge to $P(x, \cdot)$ in variation norm. Then there is a continuous bounded function $w: \mathbb{R}^{d} \times S$ $\rightarrow R$ and a constant $\lambda$ such that

$$
\begin{aligned}
& w(z, \pi)+\lambda=\sup _{\alpha \in[0,1-\Lambda], \pi^{\prime} \in S}\left[\ln h(\alpha)+\ln e\left(\pi, \pi^{\prime}, \alpha\right)\right. \\
& \left.\quad+E_{z}\left\{\ln \left(\pi^{\prime T} \zeta(z(1), \xi(1))\right)\right\}+E_{z}\left\{w\left(z(1), g\left(\pi^{\prime} \diamond \zeta(z(1), \xi(1))\right)\right)\right\}\right] .
\end{aligned}
$$

The constant $\lambda$ is an optimal value of the cost functional (14), and the strategy

$$
\left(\widehat{\alpha}\left(z(t), \pi^{-}(t)\right), \widehat{\pi}\left(z(t), \pi^{-}(t)\right)\right)
$$

where $\widehat{\alpha}$ and $\widehat{\pi}$ are Borel measurable selectors for which the supremum in (17) is attained, is optimal.

Proof. Consider first the discounted control problem, the value function $w^{\beta}$ of which is a solution to the following Bellman equation:

$$
\begin{aligned}
w^{\beta}(z, \pi)= & \sup _{\alpha \in[0,1-\Lambda], \pi^{\prime} \in S}\left[\ln h(\alpha)+\ln e\left(\pi, \pi^{\prime}, \alpha\right)\right. \\
& +E_{z}\left\{\ln \left(\pi^{\prime T} \zeta(z(1), \xi(1))\right)\right\} \\
& \left.+\beta E_{z}\left\{w^{\beta}\left(z(1), g\left(\pi^{\prime} \diamond \zeta(z(1), \xi(1))\right)\right)\right\}\right],
\end{aligned}
$$

with $\beta \in(0,1)$. We shall prove that there is a unique continuous bounded solution to (18). For a continuous bounded $v: \mathbb{R}^{d} \times S \rightarrow \mathbb{R}$ let

$$
\begin{aligned}
& T_{\beta} v(z, \pi)=\sup _{\alpha \in[0,1-\Lambda], \pi^{\prime} \in S}\left[\ln h(\alpha)+\ln e\left(\pi, \pi^{\prime}, \alpha\right)\right. \\
& \left.+E_{z}\left\{\ln \left(\pi^{\prime T} \zeta(z(1), \xi(1))\right)\right\}+\beta E_{z}\left\{v\left(z(1), g\left(\pi^{\prime} \diamond \zeta(z(1), \xi(1))\right)\right)\right\}\right] .
\end{aligned}
$$

One can easily verify that under our assumptions the operator $T_{\beta}$ is a contraction in the space of continuous bounded functions. Consequently, there is a continuous bounded function $w^{\beta}$ which is a solution to (18). Moreover 


$$
\begin{aligned}
w^{\beta}\left(z_{1}, \pi_{1}\right)- & w^{\beta}\left(z_{2}, \pi_{2}\right) \leq \sup _{\alpha \in[0,1-\Lambda], \pi^{\prime} \in S}\left(\ln \frac{e\left(\pi_{1}, \pi^{\prime}, \alpha\right)}{e\left(\pi_{2}, \pi^{\prime}, \alpha\right)}\right. \\
& +E_{z_{1}}\left\{\ln \left(\pi^{\prime T} \zeta(z(1), \xi(1))\right)\right\}-E_{z_{2}}\left\{\ln \left(\pi^{\prime T} \zeta(z(1), \xi(1))\right)\right\} \\
& +\beta\left(E_{z_{1}}\left\{w^{\beta}\left(z(1), g\left(\pi^{\prime} \diamond \zeta(z(1), \xi(1))\right)\right)\right\}\right. \\
& \left.\left.-E_{z_{2}}\left\{w^{\beta}\left(z(1), g\left(\pi^{\prime} \diamond \zeta(z(1), \xi(1))\right)\right)\right\}\right)\right) \\
= & \sup _{\pi^{\prime} \in S}\left(I\left(\pi^{\prime}\right)+I I\left(\pi^{\prime}\right)+I I I\left(\pi^{\prime}\right)\right) .
\end{aligned}
$$

By (UE),

$$
I I I\left(\pi^{\prime}\right) \leq \kappa\left\|w^{\beta}\right\|_{\mathrm{sp}},
$$

where $\left\|w^{\beta}\right\|_{\mathrm{sp}}:=\sup _{z, \pi} w^{\beta}(z, \pi)-\inf _{z, \pi} w^{\beta}(z, \pi)$, so we have

$$
\begin{aligned}
(1-\kappa)\left\|w^{\beta}\right\|_{\mathrm{sp}} \leq & \sup _{\alpha \in[0,1-\Lambda], \pi_{1}, \pi_{2}, \pi^{\prime} \in S}\left(\ln \frac{e\left(\pi_{1}, \pi^{\prime}, \alpha\right)}{e\left(\pi_{2}, \pi^{\prime}, \alpha\right)}\right. \\
& \left.+\sup _{z_{1}, z_{2} \in \mathbb{R}^{d}}\left|f\left(z_{1}, \pi^{p}\right)-f\left(z_{2}, \pi^{p}\right)\right|\right)<\infty
\end{aligned}
$$

(taking into account that by Lemma 1 the function $e$ is bounded away from 0$)$. Hence the family

$$
\left\{w^{\beta}(z, \pi)-\inf _{\widehat{z}, \widehat{\pi}} w^{\beta}(\widehat{z}, \widehat{\pi}): \beta \in(0,1)\right\}
$$

is bounded. By continuity of transition operators (in variation norm) it is also equicontinuous so that we can use the standard Ascoli-Arzelà argument (see [11]) to take a vanishing discount approach (see [8]).

3. Risk sensitive Bellman equation. In this section we shall assume that there is a probability measure $\mu$ and a positive continuous density $p\left(z, z^{\prime}\right)$ of the transition operator $P$, i.e. for $A \in \mathcal{B}\left(\mathbb{R}^{d}\right), z \in \mathbb{R}^{d}$ we have $P(z, A)=\int_{A} p\left(z, z^{\prime}\right) \mu\left(d z^{\prime}\right)$, and furthermore

$$
\sup _{z_{1}, z_{1}^{\prime}, z_{2}, z_{2}^{\prime} \in \mathbb{R}^{d}} \frac{p\left(z_{1}, z_{1}^{\prime}\right)}{p\left(z_{2}, z_{2}^{\prime}\right)}:=M<\infty .
$$

Notice that this assumption is stronger than (UE). Furthermore by Scheffe's theorem (see [12]) the transition operators are continuous in variation topology. Additionally we shall assume that there is a $\delta>0$ such that for $\gamma \in[-\delta, 0)$ the mapping $(z, \pi) \mapsto E_{z}\left\{\left(\pi^{T} \zeta(z(1), \xi(1))\right)^{\gamma}\right\}$ is bounded and continuous.

In the next theorem we show that optimal strategies for the risk sensitive cost functional (13) depend on the current value of the portfolio process $\pi^{-}(n)$ and the factor process $z(n)$ only (they do not depend on the wealth 
process and this could be already noticed from the form (15) of the cost functional (13)).

THEOREM 2. For $\gamma \in[-\delta, 0)$ there is a bounded continuous function $w_{\gamma}: \mathbb{R}^{d} \times S \rightarrow R$ and a constant $\lambda_{\gamma}$ such that

$$
\begin{aligned}
w_{\gamma}(z, \pi)+\gamma \lambda_{\gamma}= & \inf _{\alpha \in[0,1-\Lambda], \pi^{\prime} \in S}\left[\gamma\left(\ln h(\alpha)+\ln e\left(\pi, \pi^{\prime}, \alpha\right)\right)\right. \\
& +\ln E_{z}\left\{\operatorname { e x p } \left\{\gamma \ln \left(\pi^{\prime} \zeta(z(1), \xi(1))\right)\right.\right. \\
& \left.\left.+w_{\gamma}\left(z(1), g\left(\pi^{\prime} \diamond \zeta(z(1), \xi(1))\right)\right)\right\}\right] .
\end{aligned}
$$

Moreover $\lambda_{\gamma}$ is the optimal value of the cost functional (13), and the strategy

$$
\left(\widehat{\alpha}_{\gamma}\left(z(t), \pi^{-}(t)\right), \widehat{\pi}_{\gamma}\left(z(t), \pi^{-}(t)\right)\right),
$$

where $\widehat{\alpha}_{\gamma}$ and $\widehat{\pi}_{\gamma}$ are Borel measurable selectors for which the supremum in (22) is attained, is optimal.

Proof. We consider first a version of the risk sensitive discounted cost functional (see [3]). The value function $w^{\beta}$ corresponding to that control problem is continuous and is a solution to the following Bellman equation:

$$
\begin{aligned}
w^{\beta}(z, \pi, \gamma)= & \inf _{\alpha \in[0,1-\Lambda], \pi^{\prime} \in S}\left[\gamma\left(\ln h(\alpha)+\ln e\left(\pi, \pi^{\prime}, \alpha\right)\right)\right. \\
& +\ln E_{z}\left\{\operatorname { e x p } \left\{\gamma \ln \left(\pi^{\prime T} \zeta(z(1), \xi(1))\right)\right.\right. \\
& \left.\left.\left.+w^{\beta}\left(z(1), g\left(\pi^{\prime} \diamond \zeta(z(1), \xi(1))\right), \beta \gamma\right)\right\}\right\}\right] .
\end{aligned}
$$

Therefore by (21),

$$
\begin{gathered}
w^{\beta}\left(z_{1}, \pi_{1}, \gamma\right)-w^{\beta}\left(z_{2}, \pi_{2}, \gamma\right) \leq \sup _{\alpha \in[0,1-\Lambda], \pi^{\prime} \in S}\left(\ln \frac{e\left(\pi_{1}, \pi^{\prime}, \alpha\right)}{e\left(\pi_{2}, \pi^{\prime}, \alpha\right)}\right. \\
+\ln \frac{E_{z_{1}}\left\{\operatorname { e x p } \left\{\gamma \ln \left(\pi^{\prime T} \zeta(z(1), \xi(1))\right)\right.\right.}{E_{z_{2}}\left\{\operatorname { e x p } \left\{\gamma \ln \left(\pi^{\prime T} \zeta(z(1), \xi(1))\right)\right.\right.} \\
\left.\quad \frac{\left.\left.+w^{\beta}\left(z(1), g\left(\pi^{\prime} \diamond \zeta(z(1), \xi(1))\right), \beta \gamma\right)\right\}\right\}}{\left.\left.+w^{\beta}\left(z(1), g\left(\pi^{\prime} \diamond \zeta(z(1), \xi(1))\right), \beta \gamma\right)\right\}\right\}}\right) \\
\leq \sup _{\alpha \in[0,1-\Lambda], \pi^{\prime} \in S} \ln \frac{e\left(\pi_{1}, \pi^{\prime}, \alpha\right)}{e\left(\pi_{2}, \pi^{\prime}, \alpha\right)}+\ln M .
\end{gathered}
$$

Consequently, for fixed $\bar{z} \in \mathbb{R}^{d}$ and $\bar{\pi} \in S$ the family

$$
\left\{\bar{w}^{\beta}(z, \pi, \gamma):=w^{\beta}(z, \pi, \gamma)-w^{\beta}(\bar{z}, \bar{\pi}, \gamma): \gamma \in[-\delta, 0)\right\}
$$

is bounded, i.e. there is a constant $L$ (independent of $\gamma$ ) such that

$$
\left|\bar{w}^{\beta}(z, \pi, \gamma)\right| \leq L \text {. }
$$

Using continuity of the density of the transition operator $P$ we easily show its equicontinuity. Therefore there is a subsequence $\beta_{n} \rightarrow 1$ and a family $\bar{w}_{m}(z, \pi)$ such that $\bar{w}^{\beta_{n}}\left(z, \pi, \beta_{n}^{m-1} \gamma\right)$ converges uniformly on compact subsets 
to $\bar{w}_{m}(z, \pi)$. Moreover since by $(23)$,

$$
\begin{aligned}
w^{\beta}\left(\bar{z}, \bar{\pi}, \beta^{m-1} \gamma\right)-w^{\beta}\left(\bar{z}, \bar{\pi}, \beta^{m} \gamma\right) \leq \sup _{\alpha \in[0,1-\Lambda], \pi^{\prime} \in S}\left[\beta^{m-1} \gamma(\ln h(\alpha)\right. \\
\left.\left.+\ln e\left(\bar{\pi}, \pi^{\prime}, \alpha\right)\right)+\ln E_{\bar{z}}\left\{\exp \left\{\beta^{m-1} \gamma \ln \left(\pi^{\prime T} \zeta(z(1), \xi(1))\right)+L\right\}\right\}\right]
\end{aligned}
$$

and

$$
\begin{aligned}
& w^{\beta}\left(\bar{z}, \bar{\pi}, \beta^{m-1} \gamma\right)-w^{\beta}\left(\bar{z}, \bar{\pi}, \beta^{m} \gamma\right) \geq \inf _{\alpha \in[0,1-\Lambda], \pi^{\prime} \in S}\left[\beta^{m-1} \gamma(\ln h(\alpha)\right. \\
& \left.\left.+\ln e\left(\bar{\pi}, \pi^{\prime}, \alpha\right)\right)+\ln E_{\bar{z}}\left\{\exp \left\{\beta^{m-1} \gamma \ln \left(\pi^{\prime T} \zeta(z(1), \xi(1))\right)-L\right\}\right\}\right],
\end{aligned}
$$

for a suitably chosen subsequence $\lambda_{\gamma}\left(\beta^{m}\right):=w^{\beta}\left(\bar{z}, \bar{\pi}, \beta^{m-1} \gamma\right)-w^{\beta}\left(\bar{z}, \bar{\pi}, \beta^{m} \gamma\right)$ converges to $\lambda_{\gamma}^{m}$. The family $\left\{\bar{w}_{m}(z, \pi): m=1,2, \ldots\right\}$ is also bounded and equicontinuous and there is a subsequence such that $\bar{w}_{m}$ converges to $w_{\gamma}$, and $\lambda_{\gamma}^{m}$ to $\lambda_{\gamma}$, a solution to (22).

4. Risk sensitive asymptotics. In this section we are interested in the limit behaviour of the cost functional $J^{\gamma}$ when $\gamma$ increases to 0 . Notice first that by the Hölder and Jensen inequalities we obtain for $\gamma_{1} \leq \gamma_{2}<0$,

$$
\begin{aligned}
J_{X^{-}, z, \pi^{-}}^{\gamma_{1}}(\alpha(n), \pi(n)) & \leq J_{X^{-}, z, \pi^{-}}^{\gamma_{2}}(\alpha(n), \pi(n)) \\
& \leq J_{X^{-}, z, \pi^{-}}(\alpha(n), \pi(n)) .
\end{aligned}
$$

Assume additionally a kind of nondegeneracy for $\zeta$ :

(ND) for each $z \in \mathbb{R}^{d}$ the vector $\zeta(z, \xi(1))$ has a positive density on $(0, \infty)^{m}$. We have

Proposition 1. Under (ND) for Borel measurable functions $\bar{\alpha}: \mathbb{R}^{d} \times S$ $\rightarrow[0, \Lambda]$ and $\bar{\pi}: \mathbb{R}^{d} \times S \rightarrow S_{\theta}$, where $\theta>0$ and $S_{\theta}:=\left\{\left(\nu_{1}, \ldots, \nu_{m}\right)^{T} \in S:\right.$ $\left.\nu_{i} \geq \theta, i=1, \ldots, m\right\}$ we have

$$
\begin{aligned}
J_{X^{-}, z, \pi^{-}}^{\gamma}\left(\bar{\alpha}\left(z(n), \pi^{-}(n)\right), \bar{\pi}\left(z(n), \pi^{-}(n)\right)\right) & \\
& \rightarrow J_{X^{-}, z, \pi^{-}}\left(\bar{\alpha}\left(z(n), \pi^{-}(n)\right), \bar{\pi}\left(z(n), \pi^{-}(n)\right)\right)
\end{aligned}
$$

as $\gamma$ increases to 0 .

Proof. Since the proof is rather technical we point out the main steps only. By the assumption we imposed on the control $\bar{\pi}$ and assumption (ND) the pair $\left(z(n), \pi^{-}(n)\right)$ forms a Markov process satisfying a minorization property (see [4]) with an ergodic minorization set $C=K \times S_{\theta}$, where $K$ is a compact set in $\mathbb{R}^{d}$. Consequently, one can consider the splitting of this pair. This allows us to study a multiplicative Poisson equation as in [4] corresponding to the random terms depending on $\left(z(n), z(n+1), \pi^{-}(n), \xi(n+1)\right)$. To be more precise: let

$$
\begin{aligned}
q\left(z, z^{\prime}, \pi, \xi\right):= & \ln (h(\bar{\alpha}(z, \pi)))+\ln e(\pi, \bar{\pi}(z, \pi), \bar{\alpha}(z, \pi)) \\
& +\ln \left(\bar{\pi}(z, \pi)^{T} \zeta\left(z^{\prime}, \xi\right)\right) .
\end{aligned}
$$




$$
\begin{aligned}
& \widehat{w}(x) \\
& =\ln \widehat{E}_{x}\left\{\exp \left\{\sum_{n=0}^{\tau_{C_{1}}} \gamma q\left(z(n), z(n+1), \pi^{-}(n), \xi(n+1)\right)-\lambda_{\gamma}(\bar{\alpha}, \bar{\pi})\right\}\right\}
\end{aligned}
$$

with $\widehat{E}$ corresponding to the split Markov process, $x=\left(z, \pi, x^{2}\right), x^{2} \in\{0,1\}$, $\tau_{C_{1}}$ the first hitting time of the set $C \times\{1\}$ and $\lambda_{\gamma}(\bar{\alpha}, \bar{\pi})$ the value of the risk sensitive cost functional $\gamma J^{\gamma}$ corresponding to the control functions $\bar{\alpha}, \bar{\pi}$. Then $\widehat{w}$ is a solution to the Poisson equation

$$
\begin{aligned}
e^{w\left(z, \pi, x^{2}\right)}=\widehat{E}_{z}\{\exp \{\gamma q(z, z(1), \pi, \xi(1)) & -\lambda_{\gamma}(\bar{\alpha}, \bar{\pi}) \\
& \left.\left.+w\left(z(1), \pi(1), x^{2}(1)\right)\right\}\right\} .
\end{aligned}
$$

Finally, we prove a version of Proposition 3 of [6], which shows the convergence of the solutions to the multiplicative Poisson equation (29) to the solution of an additive Poisson equation and this way implies the convergence (26).

REMARK 1. An alternative approach based on the large deviation principle as in [5] gives the convergence in (26) for continuous functions $\bar{\alpha}$ and $\bar{\pi}$ only and requires additional assumptions. Notice that assumption (ND) is rather strong and we in fact need only the existence of an ergodic minorization set, as was pointed out in the proof of Proposition 1.

We can now summarize the above results (cf. Theorem 3 of [5]):

COROLlaRY 1. If an optimal control $\widehat{\pi}$ to the risk neutral problem does not allow eliminating investments in any of the assets, i.e. there is a positive $\theta$ such that $\widehat{\pi}_{i}\left(z, \pi^{p}\right) \geq \theta$ for $z \in \mathbb{R}^{d}$ and $\pi^{p} \in S$, then under (ND), $\lambda_{\gamma} \rightarrow \lambda$ as $\gamma$ increases to 0 . Furthermore an optimal control for the risk neutral cost functional is nearly optimal for the risk sensitive functional when $\gamma$ is close to 0 .

5. Remarks on assumptions and further extensions. Notice first that we used a very nice ergodic structure of the factor process $(z(n))$ (see assumptions (UE) and then (21)) for clarity of the assumptions and presentation. Using the methodology of the papers [4] and [5] under some assumptions we can extend the result to the case when $(z(n))$ is a Markov process with the minorization property. A further extension to the case when the factors depend on the same disturbances, e.g., when $z(n+1)=r(z(n), \xi(n+1))$, is nontrivial. When we allow consumption and impose an assumption concerning diversification of portfolio (see [13]) we can use some arguments of the paper [13]. The general case without this assumption requires additional technicalities that go beyond the scope of this paper. Notice moreover that it 
was crucial for our approach that consumption rate $\alpha$ was not greater than $1-\Lambda$, since by Lemma 1 we were allowed to choose (after transactions) any portfolio $\pi^{\prime} \in S$. The case without this assumption is more complicated and Theorems 1 and 2 may not be true.

\section{References}

[1] T. R. Bielecki and S. Pliska, Risk-sensitive dynamic asset management, Appl. Math. Optim. 39 (1999), 337-360.

[2] - - - Risk sensitive dynamic asset management in the presence of transaction costs, Finance Stoch. 4 (2000), 1-33.

[3] G. B. Di Masi and Ł. Stettner, Risk sensitive control of discrete time Markov processes with infinite horizon, SIAM J. Control Optim. 38 (2000), 61-78.

[4] -, -, Infinite horizon risk sensitive control of discrete time Markov processes under minorization property, ibid., submitted.

[5] - - - Remarks on risk neutral and risk sensitive portfolio optimization, in: Proc. Conference in honour of A. Shiryaev, to appear.

[6] -, - On additive and multiplicative (controlled) Poisson equations, in: Approximation and Probability, Banach Center Publ. 72, to appear.

[7] W. H. Fleming and S. J. Sheu, Risk sensitive control and an optimal investment (II), Ann. Appl. Probab. 12 (2002), 730-767.

[8] O. Hernandez-Lerma and J. B. Lasserre, Discrete-Time Markov Control Processes, Springer, 1996.

[9] G. Iyengar, Discrete time growth optimal investment with costs, preprint.

[10] H. Nagai, Optimal strategies for risk sensitive portfolio optimization problems for general factor models, SIAM J. Control Optim. 41 (2003), 1779-1800.

[11] H. L. Royden, Real Analysis, Mac Millan, New York, 1968.

[12] H. Scheffe, A useful convergence theorem for probability distributions, Ann. Math. Statist. 18 (1947), 434-438.

[13] E. Stettner, Risk sensitive portfolio optimization, Math. Meth. Oper. Res. 50 (1999), 463-474.

Institute of Mathematics

Polish Academy of Sciences

Śniadeckich 8

00-956 Warszawa, Poland

E-mail: stettner@impan.gov.pl

Received on 13.12.2005;

revised version on 28.12.2005 